\title{
Hairpin RNAs derived from RNA polymerase II and polymerase III promoter-directed transgenes are processed differently in plants
}

\author{
MING-BO WANG, CHRISTOPHER A. HELLIWELL, LI-MIN WU, PETER M. WATERHOUSE, \\ W. JAMES PEACOCK, and ELIZABETH S. DENNIS \\ Commonwealth Scientific and Industrial Research Organisation Plant Industry, Canberra, Australian Capital Territory 2601, Australia
}

\begin{abstract}
RNA polymerase III (Pol III) as well as Pol II (35S) promoters are able to drive hairpin RNA (hpRNA) expression and induce target gene silencing in plants. siRNAs of 21 nt are the predominant species in a 35S Pol II line, whereas 24- and/or 22nucleotide (nt) siRNAs are produced by a Pol III line. The $35 \mathrm{~S}$ line accumulated the loop of the hpRNA, in contrast to full-length hpRNA in the Pol III line. These suggest that Pol II and Pol III-transcribed hpRNAs are processed by different pathways. One Pol III transgene produced only 24-nt siRNAs but silenced the target gene efficiently, indicating that the 24-nt siRNAs can direct mRNA degradation; specific cleavage was confirmed by 5' rapid amplification of cDNA ends (RACE). Both Pol II- and Pol IIIdirected hpRNA transgenes induced cytosine methylation in the target DNA. The extent of methylation is not correlated with the level of 21-nt siRNAs, suggesting that they are not effective inducers of DNA methylation. The promoter of a U6 transgene was significantly methylated, whereas the promoter of the endogenous U6 gene was almost free of cytosine methylation, suggesting that endogenous sequences are more resistant to de novo DNA methylation than are transgene constructs.
\end{abstract}

Keywords: gene silencing; siRNA; RNA polymerase III; promoter; hpRNA; RNA-directed DNA methylation

\section{INTRODUCTION}

RNA polymerase III (Pol III) promoters have been widely used to direct the expression of short hairpin RNA (hpRNA) constructs to achieve silencing of target genes in animals (Yu et al. 2002; Hannon and Rossi 2004). In plants, efficient RNA silencing has been achieved mostly with long hpRNA or artificial microRNA constructs directed by RNA polymerase II (Pol II) promoters (Watson et al. 2005; Schwab et al. 2006); Pol III promoters have been used (Lu et al. 2004) but not extensively, although they may have properties complementary to those of the Pol II promoters.

Pol III promoters control the expression of most small cytoplasmic RNAs including tRNAs and 5S rRNA, some small nuclear RNAs (snRNA), short interspersed element (SINE) retroposon RNA, and several viral RNAs (Paule and White 2000; Arnaud et al. 2001). Pol III promoters can be

Reprint requests to: Ming-Bo Wang, Commonwealth Scientific and Industrial Research Organisation Plant Industry, P.O. Box 1600, Canberra, Australian Capital Territory 2601, Australia; e-mail: ming-bo.wang@ csiro.au; fax: 610262465000.

Article published online ahead of print. Article and publication date are at http://www.rnajournal.org/cgi/doi/10.1261/rna.760908. categorized into four types according to the position of transcriptional regulatory elements (Paule and White 2000; Arnaud et al. 2001). The sequence elements required for the activity of type-1, -2 , and -4 promoters are located both within and outside the coding region; type- 3 promoters contain all the regulatory elements upstream of the transcriptional start site and can therefore be readily adapted for directing expression of heterologous RNAs. Pol IIImediated transcription terminates at a sequence with four or more consecutive thymine $(\mathrm{T})$ residues (Gunnery et al. 1999; Paule and White 2000), producing a defined 3' terminus. Pol II-mediated transcription usually generates RNA with a long 3' untranslated sequence and a poly(A) tail. Many Pol III-transcribed RNAs, such as snRNAs, are localized and function in the nucleus. In contrast, Pol IItranscribed RNA is usually exported to the cytoplasm.

We tested several plant type-3 Pol III promoters and compared them with the $35 \mathrm{~S}$ ( $\mathrm{Pol}$ II) promoter for silencing efficiency. We found that these Pol III promoters can induce efficient silencing, that hpRNAs expressed from Pol II and Pol III promoters are processed differently, and that 24-nucteoltide (nt) siRNAs can guide mRNA cleavage in plants. 


\section{RESULTS}

\section{Pol III promoters are functional in directing silencing constructs}

Testing the promoters using a $\beta$-glucuronidase (GUS) transgene as target

Eight Pol III promoter fragments were tested (Table 1), including three $(\mathrm{AtU} 3 \mathrm{~B}+136$, AtU6 + 20, and At7SL + 86) that contain upstream motifs of the RNA-coding region. Each fragment contained the "TATA" box and an upstream sequence element (USE) typical of plant Pol III promoters (Fig. 1A). The spacing between the TATA box and USE (24 or 26 base pairs [bp]) clearly differentiates these Pol III promoters from the Pol II promoters that direct the expression of the U1, U2, U4, and U5 snRNAs, which have a TATA-USE spacing of 32-36 bp (Waibel and Filipowicz 1990). The TATA box and USE of the rice U3 promoter diverge from those of the dicot Pol III promoters, and the rice promoter contains two $\mathrm{G}+\mathrm{C}$-rich monocotspecific promoter (MSP) elements (GGCCCA) (Connelly et al. 1994) arranged as an inverted repeat $(-100 /-111)$.

A series of inverted-repeat constructs encoding hpRNA with a 93-bp stem and a 93-nt loop targeting the GUS gene were used to test the activity of these promoters. These constructs, driven either by the $35 \mathrm{~S}$ or the Pol III promoters (Fig. 1B,C), were transformed into tobacco and Arabidopsis thaliana lines expressing a 35S-GUS target gene. The level of GUS expression was reduced in the majority of the independent transformants in comparison with the untransformed controls (Fig. 2A,B); the Pol III promoters were active in both tobacco and Arabidopsis. The RNA motif-containing constructs AtU3 + 136, AtU6 + 20, and At7SL +86 did not confer more efficient silencing than the respective RNA motif-free constructs AtU3, AtU6, and At7SL (Fig. 2A,B), suggesting that these RNA sequences are not required for the activity of Pol III promoters.

The 35S-GUShp93 and OsU3-GUShp93 constructs conferred strong GUS silencing in rice (Fig. 2C). In contrast, the AtU3-GUShp93, AtU6-GUShp93, and LlU3-GUShp93 constructs gave little or no silencing; some lines that did show significant GUS silencing could have had integration of the silencing constructs near endogenous promoters that generated transcription of the GUS hpRNA. The results showed that the dicot Pol III promoters do not function in rice, consistent with the absence of the MSP element (Connelly et al. 1994).

\section{A Pol III promoter directs efficient silencing to an endogenous gene}

The Pol III (AtU6) promoter was tested against an endogenous gene, the Arabidopsis phytoene desaturase gene (PDS). AtU6- and 35S-directed constructs were designed to express hpRNA with a 42-bp dsRNA stem and a 9-nt loop targeting PDS. Almost all lines (43 out of 45) transformed with U6-PDShp42 showed high degrees of PDS silencing, indicated by intense bleaching of cotyledons and young leaves (Fig. 3A). In contrast, a much smaller proportion of the 35S-PDShp42 lines (26 of 43) showed PDS silencing; the levels of silencing in these lines were in general lower than in the U6 lines. This difference in PDS silencing was more clearly manifest in the T2 population: 18 of the $21 \mathrm{~T} 2$ U6-PDShp42 lines tested showed intermediate to strong PDS silencing (partial to complete bleaching of cotyledons and young leaves), whereas only 5 of the 19 T2 35SPDShp42 lines showed visible PDS silencing (leaf yellowing, leaf-tip bleaching, or cotyledon bleaching only) (Fig. 3B,C; data not shown).

\section{Pol II- and Pol III-transcribed hpRNAs are differentially processed}

A subset of the 35S-GUShp93 and AtU6 + 20-GUShp93 (referred to as U6-GUShp93 hereafter) Arabidopsis lines were analyzed by DNA and RNA gel blots (Fig. 4A-G). siRNAs were detected in both the 35S-GUShp93 and the U6-GUShp93 lines (Fig. 4C), indicating that the hpRNA was transcribed and processed by one or more of the four DICER-LIKE (DCL) proteins required for siRNA biogenesis in Arabidopsis (Gasciolli et al. 2005). The size pattern of

TABLE 1. The Arabidopsis, tomato, and rice PollII promoters used in this study

\begin{tabular}{llcl}
\hline $\begin{array}{l}\text { Small } \\
\text { RNAs }\end{array}$ & $\begin{array}{c}\text { Working names } \\
\text { of the promoters }\end{array}$ & Size (bp) & \multicolumn{1}{c}{ Plant species } \\
\hline 7SL-2 & At7SL & 343 & Arabidopsis (Arabidopsis thaliana Landsberg erecta) \\
& At7SL $+86^{\mathrm{b}}$ & 432 & Arabidopsis (Arabidopsis thaliana Landsberg erecta) \\
U3B & AtU3B & 334 & \\
& AtU3B $+136^{\mathrm{b}}$ & 467 & Arabidopsis (Arabidopsis thaliana Landsberg erecta) \\
U3 26 & AtU6 & 456 & Rice (Oryza sativa indica IR36) \\
U3 & AtU6 $+20^{\mathrm{b}}$ & 488 & Tomato (Lycopersicon lycopersicum "Yellow Pear") \\
\hline
\end{tabular}

${ }^{\mathrm{a}}$ Sizes include the restriction sites and the oligo (dT)s added to the PCR primers.

${ }^{b}$ Number of bases from RNA coding regions. 


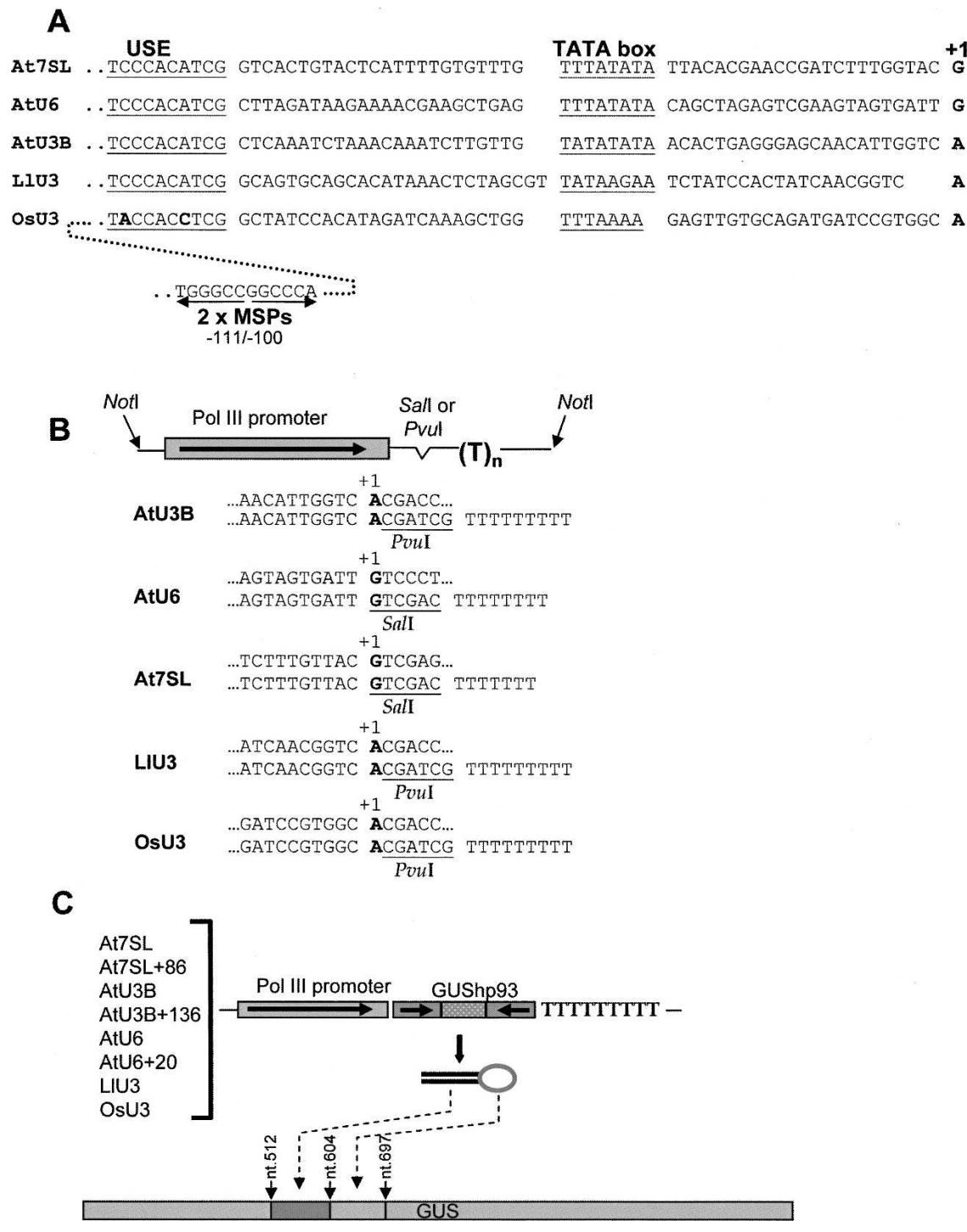

FIGURE 1. Preparation of Pol III promoter constructs. (A) Sequence comparison of the different Pol III promoters. The cis elements of the promoters, USE and TATA box, are underlined. " +1 " indicates the transcriptional start site. The underlined boldface letters indicate the two nucleotides of the rice USE that are different from those of the dicot USEs. The two divergently arranged MSP elements are shown at the bottom. (At) Arabidopsis; (Ll) tomato; (Os) rice. (B) The structure of the Pol III promoter-based intermediate vectors (top) and the sequences around the cloning sites in these intermediate vectors (bottom); the original small RNA gene sequence is shown above each of the vector sequences. The vectors contain the promoter sequence, a transcriptional termination signal of 7-9 consecutive thymines, and a restriction site (SalI or PvuI) introduced by PCR for cloning hpRNA sequences. The SalI and PvuI sites were chosen because their sequences overlap with the transcription initiation site of the small RNA genes (indicated by boldface letters). (C) The structure of the 8 Pol IIIGUShp93 constructs. The GUShp93-targeted region in the GUS ORF (relative to the ATG start codon) is indicated by the nucleotide positions (nucleotides 512-697).

the siRNAs produced differs in the $35 \mathrm{~S}$ and U6 lines (Fig. $4 \mathrm{C})$. The $35 \mathrm{~S}$ lines contained predominantly 21 -nt siRNAs, and the U6 lines had 22- and/or 24-nt siRNAs without the 21-nt class evident (Fig. 4D; Supplemental Fig. 2A). Thus, hpRNAs transcribed by the $35 \mathrm{~S}$ and the U6 promoters must be differentially processed. When gel blots of larger RNA molecules were probed with full-length GUS antisense RNA, the 35S-GUShp93 lines showed a predominant RNA species of $\sim 90 \mathrm{nt}$, whereas the U6GUShp93 lines had a predominant fragment of $\sim 300 \mathrm{nt}$ (Fig. $4 \mathrm{E}-\mathrm{G}$ ). A subset of samples were treated with RNase I (which digests only the singlestranded region in the hpRNA) and hybridized with a sense probe against the hpRNA (Fig. 4F) and an antisense probe against the loop region of the hpRNA (Fig. 4G). This showed that the two predominant fragments corresponded to the loop and full-length sequence of the GUS hpRNA in the $35 \mathrm{~S}$ and U6 lines, respectively.

The T2 PDShp42 lines (Fig. 3) were analyzed by RNA gel blot hybridization using a 21-nt DNA oligo probe complementary to the $3^{\prime}$ half of the $42-n t$ PDS sequence that forms the dsRNA stem (Fig. 5A, Probe B). The size pattern of both the siRNAs and the intermediate RNA fragments differs between the $35 \mathrm{~S}$ and the U6 lines (Fig. 5B; Supplemental Fig. 2B). The 21-nt class of siRNAs was undetectable in the U6-PDShp42 lines, which accumulated only the 24-nt siRNAs (see Supplemental Fig. 2B for determination of siRNA sizes). This was in contrast to the 35SPDShp42 lines, which contained both the 21-nt and 24-nt siRNAs. Hybridization of the same RNA gel blot with the 21-nt Probe A (Fig. 5A) detected no siRNA signals (data not shown), suggesting that the siRNAs were mostly derived from the dsRNA region near the loop-linked end in the PDShp42 RNA.

To examine whether PDS silencing in the PDShp42 lines was due to siRNAguided mRNA cleavage, $5^{\prime}$ rapid amplification of cDNA ends (RACE) was performed. The products from both the $35 \mathrm{~S}$ and the U6 lines formed a predominant band of about $220 \mathrm{bp}$ (Fig. 5C; Supplemental Fig. 2C), consistent with expected PCR products of $210-250 \mathrm{bp}$ for cleavage within the 42-nt target region. Sequencing of the $\sim 220$-bp RACE product showed cleavage predominantly between nucleotides 1597 and 1598 of the PDS mRNA in both the $35 \mathrm{~S}$ and U6 lines (Fig. 5D). This cleavage site locates in the $3^{\prime}$ region of the 42-nt target sequence, 
A

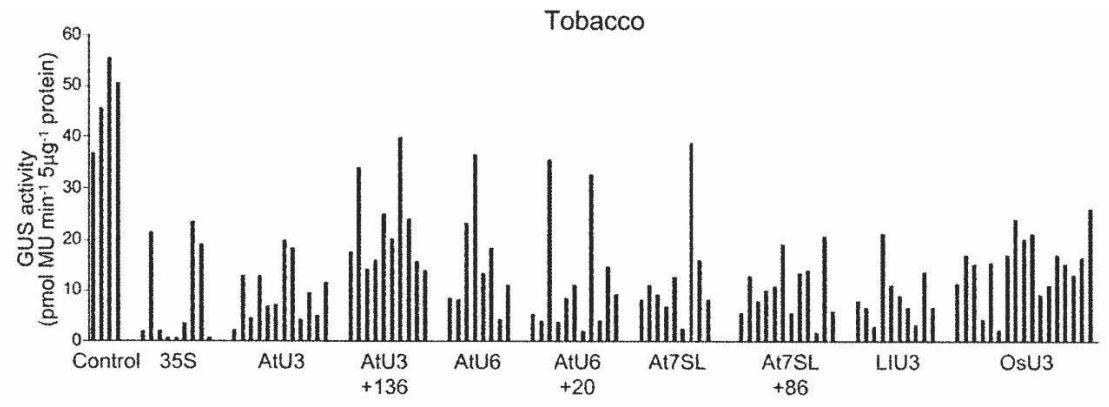

B
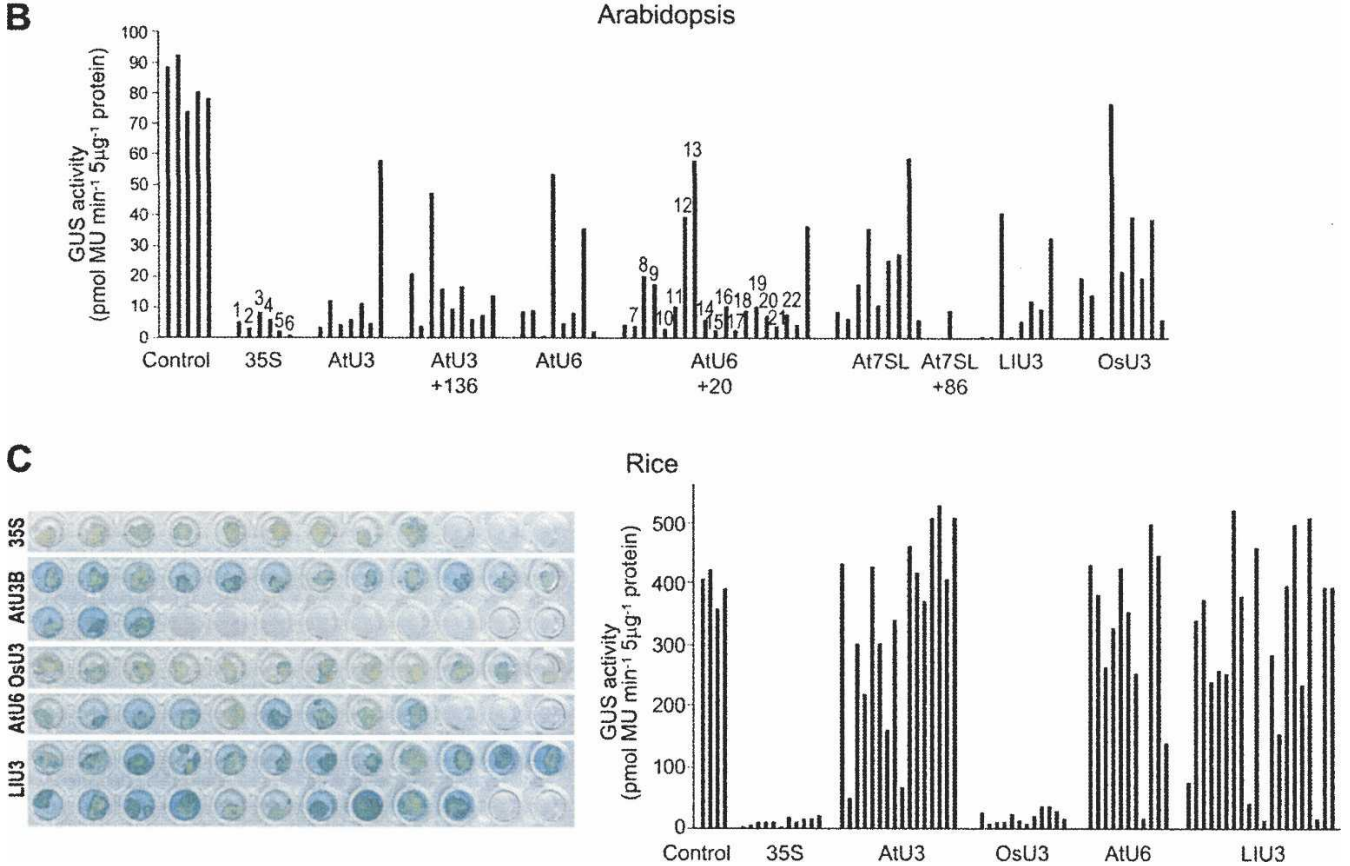

FIGURE 2. Testing of the Pol III promoters in tobacco, Arabidopsis, and rice. $(A, B)$ The Pol III promoter-directed GUShp93 constructs confer silencing against a GUS target gene in tobacco $(A)$ and Arabidopsis $(B)$. Each bar represents the GUS expression level of an independent transgenic line. The controls are sibling plants from the untransformed 35S-GUS tobacco $(A)$ or Arabidopsis $(B)$ line. The numbers above the bars for the $35 \mathrm{~S}$ and U6 +20 lines in $B$ indicate those used in subsequent DNA and RNA analysis in Fig. 4. (C) Only the rice Pol III promoter and the 35S Pol II promoter are functional in rice, while the dicotyledonous Pol III promoters show no significant activity. (Left) Assay of GUS expression by histochemical staining of transformed rice callus lines. Callus tissue in each well represents an independent line; (right) fluorimetric assay of GUS expression in transformed rice callus lines. The four controls are callus pieces derived from the previously described Ubil-GUS line (Wang and Waterhouse 2000) that was used in the transformation with the four different GUShp93 constructs.

consistent with siRNAs being derived mainly from the $3^{\prime}$ half of the 42-bp dsRNA stem.

\section{Pol III transgene-induced silencing is associated with de novo DNA methylation}

Pol II-directed hpRNA transgenes can induce de novo methylation of cytosines in target DNA sequences in the nucleus (Mette et al. 2000). To determine whether Pol IIIdirected hpRNA transgenes also induce RNA-directed DNA methylation (RdDM), we analyzed the methylation status of the target GUS sequence in the U6-GUShp93 lines \#10, \#15, and \#21. Cytosine methylation of the target GUS sequence in the 35S-GUShp93 lines \#1 and \#6 was also analyzed.
As shown in Figure 6A, cytosine methylation was almost undetectable in the target GUS sequence of the untransformed plant (NT-\#23) but was readily detected in both the 35S-GUShp93 and the U6-GUShp93 lines. The pattern of cytosine methylation in the U6 lines was similar to that in the 35S lines (Fig. 6A; Table 2). Cytosines in all sequence contexts were subject to methylation, but those in the symmetric CG and CNG contexts were more methylated than those in asymmetric contexts (Table 2), consistent with the properties of RdDM (Wang et al. 2001). As expected, methylated cytosines were more frequent in the dsRNA-targeted region than in the flanking regions, especially in the U6-GUShp93 lines (Fig. 6A; Table 2). The level of methylation in the region that corresponds to the loop 


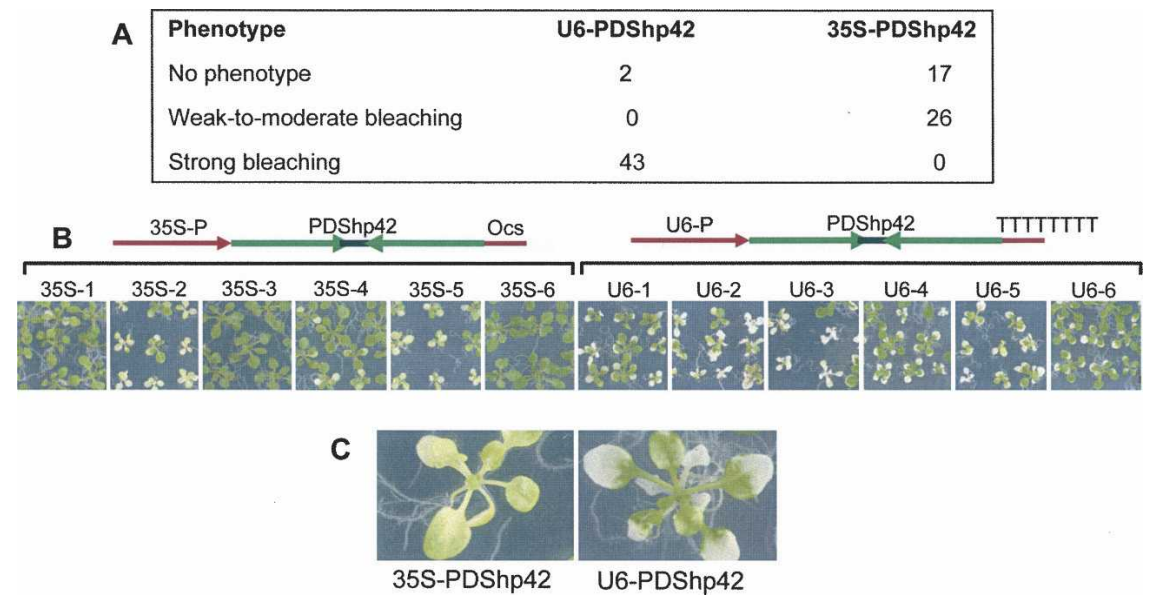

FIGURE 3. The U6-directed PDShp42 construct induces more efficient PDS silencing than the 35S-directed construct. (A) A summary of PDS silencing observed in the T1 population; (B) typical phenotypes of the T2 population; $(C)$ a close up of PDS silencing phenotypes typical of the silenced $35 \mathrm{~S}$ and U6 lines.

was also high $(15.0 \%-36.8 \%$ in the $35 \mathrm{~S}$ lines and $12.6 \%-$ $56.0 \%$ in the U6 lines); this level was much higher than in the untargeted upstream region $(4.3 \%-13.8 \%$ in the $35 \mathrm{~S}$ lines and $4.5 \%-18.2 \%$ in the U6 lines).

The promoter region of the U6-GUShp93 transgene was significantly methylated (Fig. 6B; Supplemental Fig. 3B). We examined whether the methylation of the transgene U6 promoter results in methylation of the endogenous U6 promoter. As shown in Figure 6B and Supplemental Figure $3 \mathrm{C}$, the endogenous U6 promoter was almost completely free of methylated cytosines in the two U6 lines analyzed (U6-\#15 and U6-\#21). The methylation analysis also showed that the GUS sequence of the hpRNA transgene was more methylated than that of the target GUS gene (Supplemental Fig. 4, with the exception of U6-\#21).

\section{DISCUSSION}

Our results suggest that Pol III promoters are a potentially useful alternative to Pol II promoters for directing silencing constructs in plants. In an earlier report, Lu et al. (2004) showed that the human $\mathrm{H1}$ and Arabidopsis 7SL Pol III promoters are functional in directing the silencing of a reporter gene in plants based on a short hpRNA with a 19nt dsRNA stem. We have demonstrated that a number of plant Pol III promoters are functional in directing silencing on hpRNAs with a 93-bp dsRNA stem. In addition to the silencing data for the GUS transgene, we examined the relative strength of the Pol II and Pol III promoters in directing silencing of an endogenous gene. The U6 promoter-directed hpRNA construct induced more efficient silencing than the 35S-directed construct against the endogenous PDS gene in Arabidopsis. It remains to be seen if Pol III-directed constructs are similarly effective in silencing other endogenous genes, but in a recent experi- ment, in which a small number of transgenic lines were generated, a $42-$ bp hpRNA construct directed by the AtU3B promoter also conferred more efficient silencing than a $35 \mathrm{~S}$ construct to the ethylene-insensitive gene EIN2 in Arabidopsis (data not shown).

Our RNA analyses provide strong evidence that Pol II- and Pol III-transcribed hpRNAs are processed by different sets of DCL proteins in plants. Expression of the GUS hpRNA by the 35S Pol II promoter resulted in the accumulation of predominantly 21-nt siRNAs, which indicates that the hpRNA is preferentially processed by DCL4, consistent with previous findings (Dunoyer et al. 2005; Gasciolli et al. 2005; Fusaro et al. 2006). In contrast, the Pol III lines accumulated either the 24-nt siRNA class or a mixture of 24- and 22-nt classes, with no clear presence of the 21-nt class. This suggests that the Pol III-transcribed hpRNAs are processed preferentially by DCL3 and to a lesser degree by DCL2. A recent study indicates that DCL3 acts exclusively in the nucleolus to process 24-nt heterochromatin-associated siRNAs (Pontes et al. 2006). Many of the Pol III-transcribed natural RNAs (e.g., the U6 snRNA) are known to localize in the nucleolus, and it is possible that Pol III-transcribed hpRNA is also targeted to this nuclear compartment. This may account for the preferential processing of the Pol IIItranscribed hpRNA by DCL3.

The efficient PDS silencing in the U6-PDShp42 lines containing only 24-nt siRNAs suggests that the 24-nt class is effective at guiding RNA cleavage. The RACE experiment confirms that specific mRNA cleavage does occur. It has been proposed that 21- and 22-nt siRNAs form RNA silencing complexes with ARGONAUTE 1 (AGO1) in the cytoplasm to guide mRNA cleavage, while 24-nt siRNAs interact with AGO4 in the nucleus to direct DNA methylation (Xie et al. 2004; Deleris et al. 2006). Some microRNAs (e.g., miR163) are $24 \mathrm{nt}$ in size and presumably interact with AGO1 to guide mRNA cleavage in plants (Allen et al. 2004). A recent in vitro study showed that the 24-nt siRNA-AGO4 complex possesses RNA cleavage activity (Qi et al. 2006). Whether or not the nuclear-localized siRNA-AGO4 complex can induce RNA degradation in vivo remains an interesting question, but a previous report shows that nuclear-localized RNAs can be efficiently targeted by RNA interference in animals (Robb et al. 2005).

The predominant cleavage site was identical in the 35SPDShp42 and the U6-PDShp42 plants despite the likelihood that the cleavage is guided by different size classes of siRNAs in the two plants (i.e., 21- and 24-nt in the $35 \mathrm{~S}$ plant and 24-nt in the U6 plant). One possibility is that the 
24-nt siRNAs, like the 21-nt siRNAs, also guide cleavage across nucleotides 10 and 11 of the siRNAs (Haley and Zamore 2004). A recent study showed that the 24-nt miR163 cleaves its target mRNA across nucleotides 10-11 in Arabidopsis (Allen et al. 2004).

Our results show that both Pol II- and Pol III-directed hpRNA transgenes can induce de novo cytosine methylation in plants. There was no direct correlation between the level of cytosine methylation in the GUS sequence and the abundance of the 21-nt GUS siRNAs; lines 35S-\#1 and 35S-\#6 contained abundant 21-nt siRNAs, but they were less methylated than lines U6-\#15 and U6-\#21 that did not seem to contain any 21-nt siRNAs (Figs. 4, 6A). This suggests that 21-nt siRNAs are not an effective inducer of RdDM. Among all the five lines analyzed, U6-\#21 contained the highest level of 24-nt siRNAs (Fig. 4) and showed the most intense methylation in the GUS sequence (Fig. 6A), consistent with 24-nt siRNAs being an effective inducer of RdDM. However, the GUS sequence targeted by the loop of the GUShp93 RNA was also strongly methylated (Fig. 6A; Supplemental Fig. $3)$; this supports the view that fulllength hpRNA might be a direct inducer of $\mathrm{RdDM}$ in plants (Melquist and Bender 2003). The endogenous U6 promoter was almost completely unmethylated despite the methylation of the U6 promoter in the U6-GUShp93 transgene. Consistent with this, previous studies have shown that endogenous sequences are less susceptible to silencingassociated DNA methylation than transgene sequences in plants (Stam et al. 1998; Jones et al. 1999; Vaistij et al. 2002). The GUShp93 transgenes were significantly more methylated than the target GUS gene in both the $35 \mathrm{~S}$ and U6 lines (Supplemental Fig. 4). A possible explanation for this is that the stably expressed target GUS gene, although initially introduced as a transgene, may have been "endogenized" and therefore become less susceptible to de novo methylation than the newly introduced GUShp93 transgenes.

\section{MATERIALS AND METHODS}

\section{Preparation of plasmids}

Isolation of the Pol III promoters

The promoter fragments of five small RNA genes were amplified by polymerase chain reaction (PCR): U3B, U6-26, and 7SL-2 from Arabidopsis, and U3 from tomato and rice (Table 1; for PCR primers, see Supplemental Data 1). The PCR fragments were cloned into either pGEM-T Easy (Promega) (for At7SL, At7SL + 86, AtU6, and AtU6 + 20) or pShuttle (Wang et al. 1998) (for AtU3B, AtU3B + 136, LlU3, and OsU3) and characterized by DNA sequencing using the BigDye Terminator v3.1 Cycle Sequencing Kit (Applied Biosystems) (Supplemental Data 2).

\section{Construction of the intermediate Pol III promoter vectors}

The AtU3B + 136 clone in pShuttle was used directly as the intermediate vector for cloning hpRNA sequences. AtU6 was cloned as an XhoI fragment from pGEM-T Easy into the SalI site of pShuttle, making the AtU6 intermediate vector. AtU6 + 20 was first cloned as an XhoI fragment into pShuttle at the SalI site, then as a NotI fragment from pShuttle into the NotI site of a modified $\mathrm{pBC}$ vector (Stratagene) in which the PvuI site had been removed by treatment with $P f u$ DNA polymerase followed by self-religation.
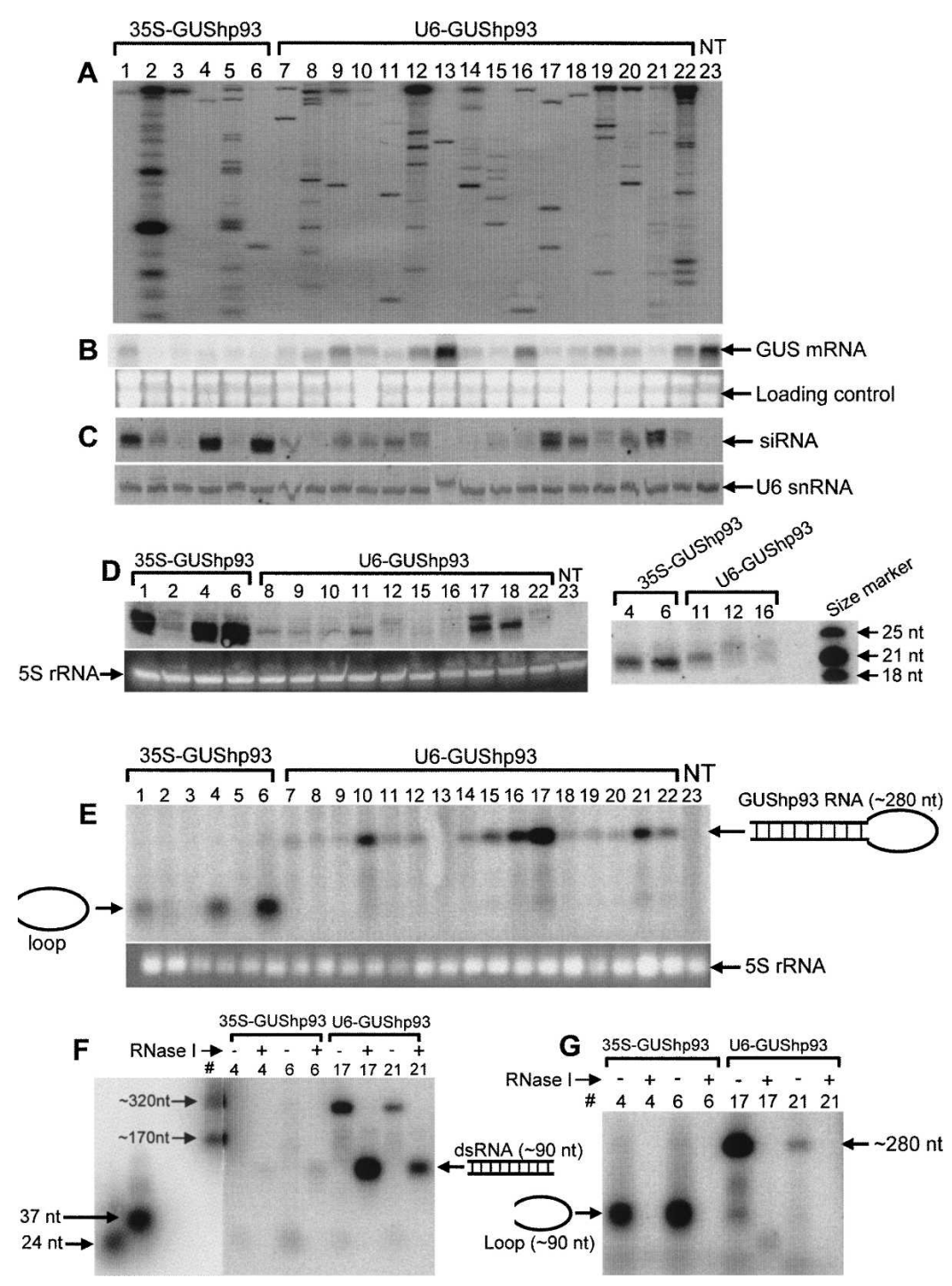

FIGURE 4. (Legend on next page) 
To prepare the AtU3B, LlU3, and OsU3 intermediate vectors, the pShuttle with HindIII and BamHI and inserted into the same sites of the modified pBC vector. Similarly, the At7SL and At7SL +86 vectors were constructed by excising the promoter fragments from their pShuttle clones with Bsp120I and SpeI and inserting into the modified $\mathrm{pBC}$ vector at the same sites.

\section{Construction of GUShp93 and PDShp42 vectors}

To assemble the inverted-repeat sequence for GUShp93, the GUS coding sequence from nucleotide 512 to nucleotide 697 (with respect to the " $A$ " residue of the translational start codon "ATG") was amplified by PCR using GUSPol3-1 and GUSPol3-2 as primers (Supplemental Data 1), digested with SalI and BamHI, and inserted into pJKK (Kirschman and Cramer 1988) at the same sites, forming pMBW448. This GUS sequence was selected as it is free of any runs of four consecutive $\mathrm{T}$ or A residues to avoid premature termination of Pol III promoter-directed transcription. The GUS sequence from nucleotide 512 to nucleotide 604, corresponding to the dsRNA stem, was PCR-amplified using the GUSPol3-1 and GUSPol3-4 primers (Supplemental Data 1), digested with BamHI and EcoRI, and inserted into pMBW448 at the same sites, giving the invertedrepeat sequence of GUShp93 (see Supplemental Data 3 for the sequence). GUShp93 was cloned as a SalI or PvuI fragment into the various intermediate Pol III vectors at the SalI, XhoI, or PvuI site to produce the Pol III-GUShp93 constructs. The 35S-GUShp93 construct was made by inserting the SalI-digested GUShp93 fragment into the XhoI site of pART7 (Gleave 1992). For Agrobacteriumpromoter fragments were excised from their respective clones in

mediated plant transformation, the Pol III-GUShp93 and 35S-GUShp93 cassettes were excised with NotI and inserted into the NotI site of the pWBVec4a vector (Wang et al. 1998).

To make the U6-PDShp42 and 35S-PDShp42 constructs, two complementary oligonucleotides (see Supplemental Data 3 for the sense-strand sequence) were annealed and ligated into the SalI site of the AtU6 intermediate vector or the XhoI site of pART7. The cassettes were then transferred as NotI fragments into pART27 (Gleave 1992) for Agrobacterium-mediated transformation.

The GUShp93 constructs were introduced into Agrobacterium tumefaciens AGLI, and the PDShp42 constructs into A. tumefaciens GV3101.

\section{Plant transformation}

Agrobacterium-mediated transformation of Arabidopsis thaliana C24 (with or without a strongly expressed 35S-GUS gene) was performed using the "floral dip" method as previously described (Clough and Bent 1998). Seed collected from the Agrobacteriuminfected plants was sterilized with chlorine gas and plated on Murashige and Skoog (MS) medium containing $10 \mathrm{mg} / \mathrm{L}$ PPT (phosphinothricin) (for pWBVec4a-based constructs) or $50 \mathrm{mg} / \mathrm{L}$ kanamycin (for pART27-based constructs) plus $100 \mathrm{mg} / \mathrm{L}$ timentin (to inhibit Agrobacterium growth). The resulting PPT- or kanamycin-resistant T1 plants were transferred to fresh MS medium for further growth before being planted in soil to set seed. A tobacco (Nicotiana tobacum Wisconsin 38) line containing a strongly expressed 35S-GUS transgene was transformed with the GUShp93 constructs using the leaf disc method as described previously (Wang et al. 2001), with PPT $(15 \mathrm{mg} / \mathrm{L})$ as the selective agent. Plants with established roots were transferred to the glasshouse. Two previously used rice lines, expressing a GUS gene (Wang and Waterhouse 2000), were transformed with the GUShp93 constructs as previously described using bialaphos as the selective agent (Wang and Waterhouse 2000). Uniformly transformed callus lines were used for GUS expression analysis. hybridized with ${ }^{32} \mathrm{P}$-labeled antisense RNA corresponding to the stem and loop regions in the GUShp93 sequence (nucleotides 512-697 of the GUS ORF). (Lower panel) U6 snRNA band used as loading control. $(D)$ Better separations of siRNAs. Subsets of the small RNA samples in $C$ were separated in either $15 \%$ (left) or 18\% (right) polyacrylamide gel and hybridized with the same probe as for $C$. Note that the two predominant siRNA bands in the U6 lines are larger than the predominant band in the $35 \mathrm{~S}$ lines, indicating that the former are 22 and $24 \mathrm{nt}$ in size and the latter 21 nt. (E) Gel blot analysis of intermediate RNAs in 35S-GUShp93 and U6GUShp93 Arabidopsis plants. Total small RNAs was separated in 5\% formaldehyde-agarose (NuSieve 3:1) gels and hybridized with ${ }^{32} \mathrm{P}$-labeled full-length antisense GUS RNA. (Lower panel) Ethidium bromide-stained 5S rRNA as loading control. (F) Four of the total small RNA samples shown in $E$ (lanes 4,6,17,21) were untreated $(-)$ or treated $(+)$ with RNase I, separated in $5 \%$ formaldehyde-agarose (NuSieve 3:1) gel, and hybridized with ${ }^{32} \mathrm{P}$-labeled sense RNA corresponding to the stem and loop of GUShp93 RNA (i.e., nucleotides 512-697 of GUS ORF), which should detect the full-length RNA and the dsRNA stem, but not the loop fragment, of the GUShp93 transcript. The molecular size markers shown are 24- and 37-nt antisense GUS DNA oligonucleotides and ${ }^{32} \mathrm{P}$-labeled in vitro sense transcript of a self-cleaving clone of the cereal yellow dwarf virus satellite RNA (pGEM.Sat, $\sim 170$ and 320 nt [Wang et al. 2001]). (G) The same four samples were untreated $(-)$ or treated $(+)$ with RNase I, separated in 5\% formaldehyde-agarose (NuSieve 3:1) gel, and hybridized with ${ }^{32} \mathrm{P}$-labeled antisense RNA corresponding to the loop of GUShp93 RNA (i.e., nucleotides 605-697 of GUS ORF). Note that the 35SGUShp93-derived RNA intermediate hybridized only with the antisense probes $(E$ and $G$ ) but not with the sense probe $(F)$, indicating the hybridizing band is the loop fragment. Also note that significant amounts of RNase I-resistant RNA could only be detected in the U6GUShp93 plants $(F)$.

\section{DNA and RNA analysis}

DNA, RNA of relatively large molecular size, and small RNA were isolated from T1 tobacco or T2 Arabidopsis plants using the following procedure: First, large-sized RNA was isolated from $\sim 2 \mathrm{~g}$ of tobacco leaf tissue or $\sim 1 \mathrm{~g}$ of Arabidopsis seedlings (grown on MS plates with appropriate selective agents) using the phenol extraction method as previously described (de Vries et al. 1988). After $\mathrm{LiCl}$ precipitation of the largesized RNA fraction, the remaining nucleic acids including DNA and small RNA were precipitated from the supernatant with one volume of isopropanol and used directly for DNA and intermediate RNA analysis. For siRNA detection, small RNAs were extracted 


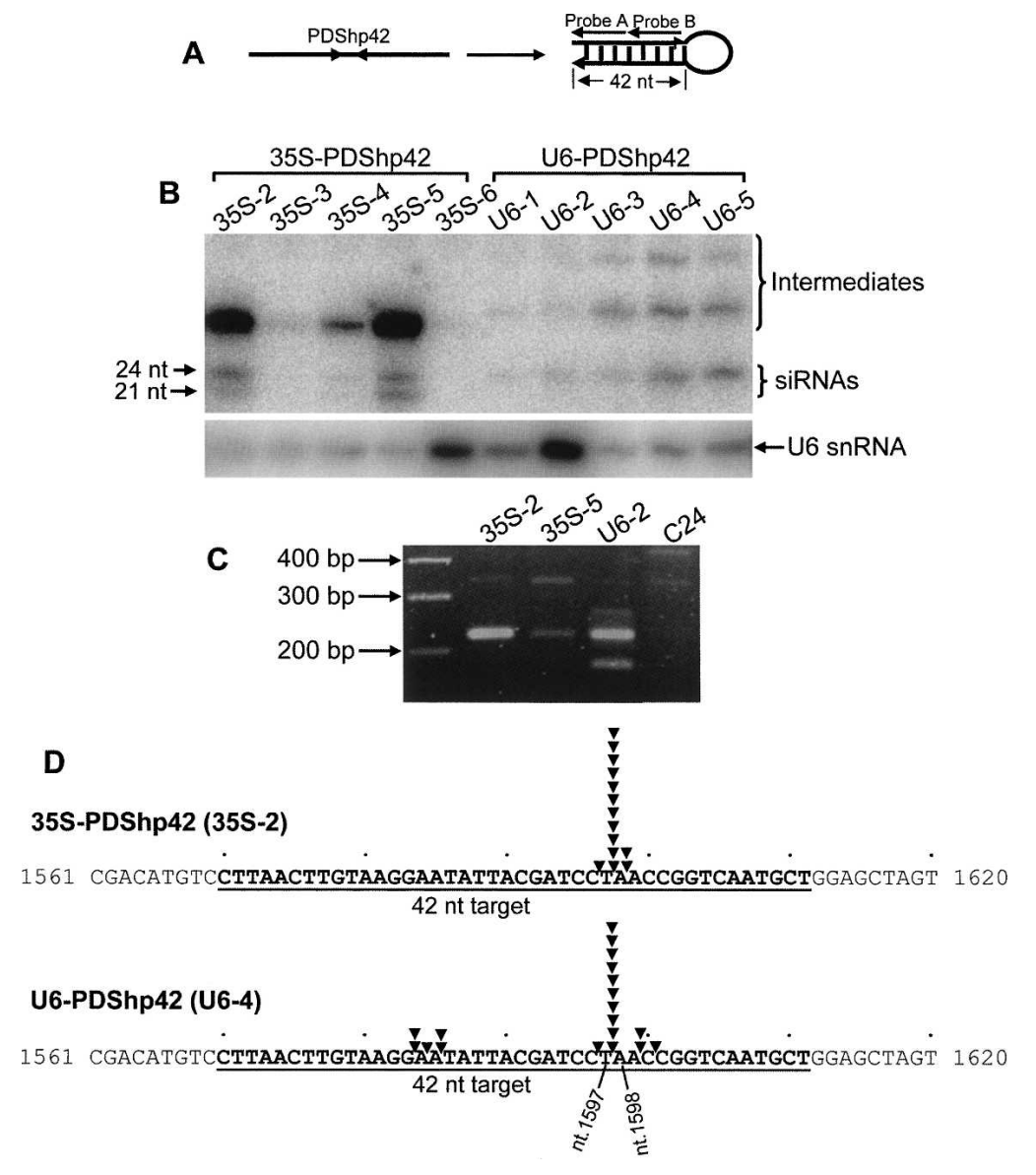

FIGURE 5. Detection of PDS siRNAs and mRNA cleavage in 35S- and U6-directed PDShp42 lines. (A) Predicted PDShp42 RNA and two DNA oligonucleotides used as probe for siRNA detection. (B) Small RNA was separated in $15 \%$ polyacrylamide gel and hybridized with the oligonucleotide Probe B $(A)$. (C) Agarose gel electrophoresis of 5' RACE-PCR products of 35SPDShp42 and U6-PDShp42 plants. C24 is an untransformed control. (D) Sequencing analysis of 5' RACE products from a 35S-PDShp42 line (35S-2) and a U6-PDShp42 line (U6-4) (Supplemental Fig. 2C). The underlined nucleotides indicate the 42-nt PDS sequence that is targeted by the PDShp42 RNA. Each arrowhead indicates one cleavage product recovered by RACE.

from the isopropanol pellet overnight at $4^{\circ} \mathrm{C}$ with $8 \mathrm{M} \mathrm{LiCl}$ and concentrated with ethanol precipitation.

For DNA gel blot analysis of the Arabidopsis lines, $\sim 10 \mu \mathrm{g}$ of each DNA/small RNA sample was digested with BamHI (80 units) (Supplemental Fig. 1) in the presence of RNase A $(5 \mu \mathrm{g})$ in $100 \mu \mathrm{L}$ volume at $37^{\circ} \mathrm{C}$ overnight. The digested DNA was purified by phenol/chloroform extraction, precipitated with $1 / 10$ volume of 3 $\mathrm{M} \mathrm{NaOAc}$ and 2.5 volumes of ethanol, and redissolved in $30 \mu \mathrm{L}$ of TE buffer. The DNA was then separated on a $0.8 \%$ agarose gel and blotted onto Hybond-N+ Nylon membrane (Amersham Pharmacia) with $0.4 \mathrm{M} \mathrm{NaOH}$. The membrane was probed with a SmaI fragment of the PPT-resistance gene from pWBVec4a (Wang et al. 1998). The hybridized membranes were washed as recommended by the manufacturer and analyzed using a PhosphorImager (FUJIFILM, FLA-5000).

To determine the target GUS mRNA levels, $\sim 30 \mu \mathrm{g}$ of each large-sized RNA preparation was separated on a $1.3 \%$ formaldehyde-agarose gel, blotted to Hybond-N Nylon membrane, UV cross-linked, and hybridized with ${ }^{32} \mathrm{P}$-labeled, full-length antisense
GUS RNA at $55^{\circ} \mathrm{C}$ in the hybridization buffer B described in the Promega "Protocols and Applications Guide." The hybridized filter was washed as recommended including RNase A treatment.

For analysis of intermediate RNAs derived from GUShp93, DNA/small RNA samples (equivalent to $\sim 120 \mu \mathrm{g}$ of large RNA) were treated with RNase-free DNase and purified with phenol/chloroform extraction and ethanol precipitation. For detecting dsRNA, the DNA/small RNA sample was further treated with RNase I to digest single-stranded RNA, followed by phenol/chloroform extraction and ethanol precipitation. The purified RNA was then separated on a $5 \%$ formaldehyde-NuSieve 3:1 agarose gel, blotted to Hybond-N Nylon membrane, UV crosslinked, and hybridized with ${ }^{32} \mathrm{P}$-labeled GUS riboprobes (as specified in Fig. 4,E-G) at $42^{\circ} \mathrm{C}$ in the hybridization buffer B described in the Promega "Protocols and Applications Guide." The filter was washed twice for $30 \mathrm{~min}$ in $2 \times \mathrm{SSC}, 0.2 \%$ SDS at $42^{\circ} \mathrm{C}$, followed by RNase A treatment for $15 \mathrm{~min}$ at room temperature $(2 \mu \mathrm{g} / \mathrm{mL}$ RNase A in $2 \times$ SSC).

For siRNA detection, small RNA samples from $8 \mathrm{M} \mathrm{LiCl}$ extraction were separated in $15 \%$ or $18 \%$ denaturing polyacrylamide gels in $1 \times$ TBE buffer and electroblotted and UV cross-linked onto Hybond-N Nylon membrane. GUS siRNAs were detected by hybridization at $38^{\circ} \mathrm{C}-42^{\circ} \mathrm{C}$ with ${ }^{32} \mathrm{P}$-labeled GUS riboprobe (as specified in the figure legends) that were pretreated with $0.2 \mathrm{M}$ sodium carbonate at $60^{\circ} \mathrm{C}$ to reduce their size to an average of $\sim 50 \mathrm{nt}$; the GUS siRNA blot was washed as for the GUShp93-derived RNA intermediates described above. The PDS siRNAs were detected by hybridization at $30^{\circ} \mathrm{C}$ using a $21-n t$ DNA oligonucleotide as specified in Figure 5 legend, and the blot was washed at room temperature with $2 \times$ SSC, $0.2 \%$ SDS.

\section{5' RACE of PDShp42 lines}

Large-sized RNA preparations from 35S-PDShp42 and U6PDShp42 plants were directly ligated with a 21-nt unphosphorylated GUS RNA oligo (5'-AACAGACGCGUGGUUACAGUC) for $1 \mathrm{~h}$ at $37^{\circ} \mathrm{C}$ using T4 RNA ligase following the manufacturer's instruction. The ligation was purified by phenol/chloroform extraction and ethanol precipitation, and used for reverse transcription using a PDS-specific reverse primer PDS-RACEr1 (5' CTTTGTAGAGGACGACATGG). PCR was first performed using a GUS DNA oligo (with identical sequence to the GUS RNA oligo used in the ligation) and PDS-RACEr1 as primers. A nested PCR was then performed using the GUS DNA oligo and PDS-RACEr2 (5'-CACAGTTTGGGATGGTCTTG) as primers. The nested PCR 


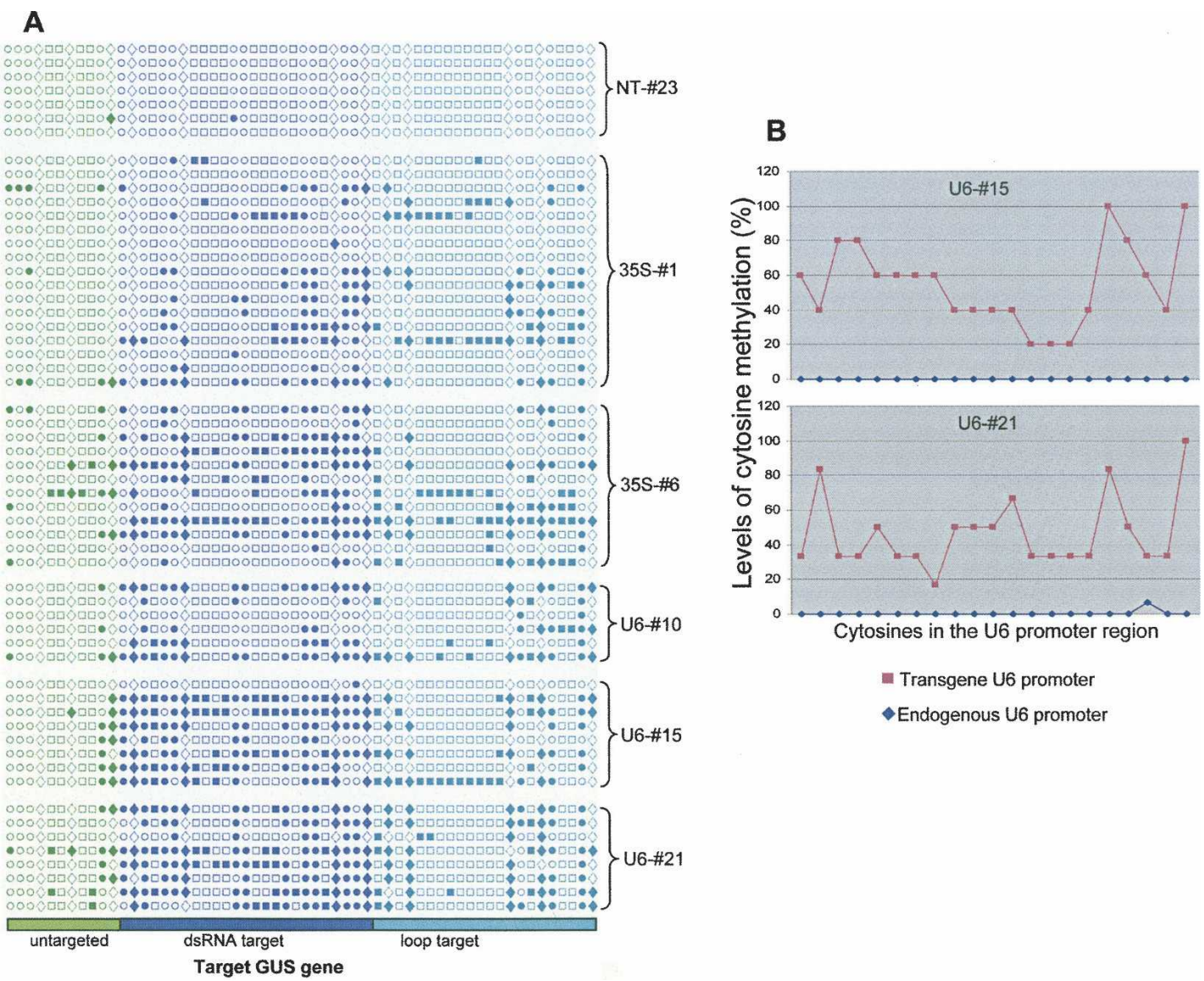

FIGURE 6. Bisulphite sequencing analysis of DNA methylation. (A) Detection of DNA methylation in the target GUS sequence. Only cytosine residues are shown, excluding those of the $5^{\prime}$ and $3^{\prime}$ sequences that overlap with the bisulphite PCR primers. Each row represents the cytosines in one bisulphite-PCR clone. The green, dark blue, and light blue symbols indicate cytosines in the untargeted upstream region, the dsRNA-targeted region, and the loop-targeted region of the target GUS sequence, respectively. The open and solid circles indicate unmethylated and methylated cytosines, respectively, in the symmetric CG context; the open and solid diamonds indicate unmethylated and methylated cytosines, respectively, in the symmetric CNG context; the open and solid rectangles indicate unmethylated and methylated cytosines, respectively, in the asymmetric context. Accession numbers on the right correspond to the same sample numbers shown in Fig. 4. (B) The promoter of the U6-GUShp93 transgene is significantly methylated (purple line), whereas the endogenous U6 promoter is almost completely free of methylation (blue line). This figure is based on the bisulphite sequencing data shown in Supplemental Fig. 3.

product was separated in agarose gel, and the $\sim 220$ band purified, cloned into pGEM-T Easy (Promega), and sequenced.

\section{Analysis of GUS expression}

GUS activity was measured using the same kinetic fluorimetric 4-methylumbelliferryl$\beta$-glucuronide (MUG) assay as previously described (Chen et al. 2005). Histochemical staining of rice callus lines to detect GUS expression was as previously described (Jefferson et al. 1987).

\section{Bisulphite sequencing}

Bisulphite treatment of Arabidopsis genomic DNA was performed following the procedure described previously (Paulin et al. 1998). Approximately $5 \mu \mathrm{g}$ of the DNA/ small RNA sample was denatured in $20 \mu \mathrm{l}$ of $0.3 \mathrm{M} \mathrm{NaOH}$ for 15 $\min$ at $37^{\circ} \mathrm{C}$. The denatured DNA was then mixed with $208 \mu \mathrm{l}$ of 6.24 M urea and $2 \mathrm{M}$ sodium metabisulphite and $12 \mu \mathrm{l}$ of freshly prepared $10 \mathrm{mM}$ hydroquinone, and the mixture was incubated in

TABLE 2. A summary of cytosine methylation detected by bisulphite genomic sequencing

\begin{tabular}{|c|c|c|c|c|c|}
\hline \multirow{3}{*}{$\frac{\text { Transgenic lines }}{\text { 35S-GUShp93 }}$} & \multirow{2}{*}{\multicolumn{2}{|c|}{ Target sequence }} & \multicolumn{3}{|c|}{ Percent methylated cytosines ${ }^{a}$} \\
\hline & & & \multirow{2}{*}{$\frac{\text { CG }(\%)}{13.8}$} & \multirow{2}{*}{$\frac{\mathrm{CNG}(\%)}{5.2}$} & \multirow{2}{*}{$\frac{\text { Other C }(\%)}{4.3}$} \\
\hline & GUS & $5^{\prime}$ region & & & \\
\hline & & dsRNA target & 40.8 & 31.9 & 12.4 \\
\hline & & Loop region & 36.8 & 25.5 & 15.0 \\
\hline \multirow[t]{3}{*}{ U6-GUShp93 } & GUS & $5^{\prime}$ region & 15.9 & 18.2 & 4.5 \\
\hline & & dsRNA target & 71.9 & 63.6 & 25.4 \\
\hline & & Loop region & 56.0 & 52.7 & 12.6 \\
\hline
\end{tabular}

${ }^{a}$ Figures indicate the percentage of methylated cytosines in the total number of cytosine residues shown in Fig. $6 \mathrm{~A}$ in the specified target regions and sequence contexts. 
a thermocycler with 20 cycles of $55^{\circ} \mathrm{C}$ for $15 \mathrm{~min}$ and $95^{\circ} \mathrm{C}$ for $30 \mathrm{sec}$. The treated DNA was then purified and treated with $\mathrm{NaOH}$ following the procedure as described by Wang et al. (2001). The following PCR primer pairs were used to amplify the lower strand of the target GUS sequence, the sequence of 35SGUShp93 and AtU6-GUShp93 transgenes, and the AtU6 promoter sequence:

Low-GUS-F $\quad\left(5^{\prime}\right.$-CTATCCCACCAAAAATAATAATTACC-3') plus Low-GUS-R (5'-TTGTTTAGTTGTAATTATTTGTTG-3') (for target GUS sequence);

Low-35S-F (5' - ATTACAATAAAAAAAAAACTATCATTCAA-3') plus Low-GUS-R (for 35S-GUShp93);

Low-U6-F (5'-TTTTTTATTTGGAGTTTTTGTAT-3') plus LowGUS-R (for AtU6-GUShp93);

Low-U6-F2 (5'-CCCATTTAAATTAAAAACAATCTTC-3') plus Low-U6-R1 (5'-TGTTGAAGAATAGAGGAAGAAGAAAT-3'); or

Low-U6-R2 (5' - GAATTATATTATTTGAGATTTTTTTTAGGT-3') (for endogenous U6 promoter).

To minimize biased amplification between methylated and unmethylated DNA, the sequences of the primers were selected such that the $3^{\prime}$-most 5-13 nt corresponded to GUS or AtU6 sequences that have no cytosines. The PCR conditions were as previously described (Wang et al. 2001). PCR products were cloned into pGEM-T Easy and sequenced using the BigDye Terminator v3.1 Cycle Sequencing Kit.

\section{SUPPLEMENTAL DATA}

Supplemental material can be found at http://www.rnajournal.org.

\section{ACKNOWLEDGMENTS}

We thank Lina Ma, Xueqin Wang, Sue Allen, and Anna Wielopolska for technical assistance; Candice Sheldon and Rudy Dolferus for providing the seed of the GUS-expressing Arabidopsis line; Carl Davies for photography; and Bill Taylor, Tony Arioli, and Michael Metzlaff for support.

Received July 31, 2007; accepted February 6, 2008.

\section{REFERENCES}

Allen, E., Xie, Z., Gustafson, A.M., Sung, G.H., Spatafora, J.W., and Carrington, J.C. 2004. Evolution of microRNA genes by inverted duplication of target gene sequences in Arabidopsis thaliana. Nat. Genet. 36: 1245-1246.

Arnaud, P., Yukawa, Y., Lavie, L., Pélissier, T., Sugiura, M., and Deragon, J.-M. 2001. Analysis of the SINE S1 Pol III promoter from Brassica; impact of methylation and influence of external sequences. Plant J. 26: 295-305.

Chen, S., Helliwell, C.A., Wu, L.M., Dennis, E.S., Upadhyaya, N.M., Zhang, R., Waterhouse, P.M., and Wang, M.B. 2005. A novel TDNA vector design for selection of transgenic lines with simple transgene integration and stable transgene expression. Funct. Plant Biol. 32: 671-681.

Clough, S.J. and Bent, A.F. 1998. Floral dip: A simplified method for Agrobacterium-mediated transformation of Arabidopsis thaliana. Plant J. 16: 735-743.
Connelly, S., Marshallsay, C., Leader, D., Brown, J.W.S., and Filipowicz, W. 1994. Small nuclear RNA genes transcribed by either RNA polymerase II or RNA polymerase III in monocot plants share three promoter elements and use a strategy to regulate gene expression different from that used by their dicot plant counterparts. Mol. Cell. Biol. 14: 5910-5919.

de Vries, S., Hoge, H., and Bisseling, T. 1988. Isolation of total and polysomal RNA from plant tissues. In Plant molecular biology manual (eds. S.B. Gelvin et al.), pp. B6/1-B6/13. Kluwer Academic Publishers, Dordrecht, The Netherlands.

Deleris, A., Gallego-Bartolome, J., Bao, J., Kasschau, K.D., Carrington, J.C., and Voinnet, O. 2006. Hierarchical action and inhibition of plant Dicer-like proteins in antiviral defense. Science 313: $68-71$.

Dunoyer, P., Himber, C., and Voinnet, O. 2005. DICER-LIKE 4 is required for RNA interference and produces the 21-nucleotide small interfering RNA component of the plant cell-to-cell silencing signal. Nat. Genet. 37: 1356-1360.

Fusaro, A.F., Matthew, L., Smith, N.A., Curtin, S.J., Dedic-Hagan, J., Ellacott, G.A., Watson, J.M., Wang, M.B., Brosnan, C., Carroll, B.J., et al. 2006. RNA interference-inducing hairpin RNAs in plants act through the viral defence pathway. EMBO Rep. 7: $1168-1175$.

Gasciolli, V., Mallory, A.C., Bartel, D.P., and Vaucheret, H. 2005. Partially redundant functions of Arabidopsis DICER-like enzymes and a role for DCL4 in producing trans-acting siRNAs. Curr. Biol. 15: $1-7$.

Gleave, A.P. 1992. A versatile binary vector system with a T-DNA organisational structure conducive to efficient integration of cloned DNA into the plant genome. Plant Mol. Biol. 20: 1203-1207.

Gunnery, S., Ma, Y., and Mathews, M.B. 1999. Termination sequence requirements vary among genes transcribed by RNA polymerase III. J. Mol. Biol. 286: 745-757.

Haley, B. and Zamore, P.D. 2004. Kinetic analysis of the RNAi enzyme complex. Nat. Struct. Mol. Biol. 11: 599-606.

Hannon, G.J. and Rossi, J.J. 2004. Unlocking the potential of the human genome with RNA interference. Nature 431: 371-378.

Jefferson, R.A., Kavanagh, T.A., and Bevan, M.W. 1987. GUS fusion: $\beta$-glucuronidase as a sensitive and versatile gene fusion marker in higher plants. EMBO J. 6: 3901-3907.

Jones, L., Hamilton, A.J., Voinnet, O., Thomas, C.L., Maule, A.J., and Baulcombe, D.C. 1999. RNA-DNA interactions and DNA methylation in post-transcriptional gene silencing. Plant Cell 11: 2291-2301.

Kirschman, J.A. and Cramer, J.H. 1988. Two new tools: Multipurpose cloning vectors that carry kanamycin or spectinomycin/streptomycin resistance markers. Gene 68: 163-165.

Lu, S., Shi, R., Tsao, C.C., Yi, X., Li, L., and Chiang, V.L. 2004. RNA silencing in plants by the expression of siRNA duplexes. Nucleic Acids Res. 32: e171. doi: 10.1093/nar/gnh170.

Melquist, S. and Bender, J. 2003. Transcription from an upstream promoter controls methylation signalling from an inverted repeat of endogenous genes in Arabidopsis. Genes \& Dev. 17: 2036-2047.

Mette, M.F., Aufsatz, W., van der Winden, J., Matzke, M.A., and Matzke, A.J.M. 2000. Transcriptional silencing and promoter methylation triggered by double-stranded RNA. EMBO J. 19: 5194-5201.

Paule, M.R. and White, R.J. 2000. Transcription by RNA polymerase I and III. Nucleic Acids Res. 28: 1283-1298. doi: 10.1093/nar/ 28.6.1283.

Paulin, R., Grigg, G.W., Davey, M.W., and Piper, A.A. 1998. Urea improves efficiency of bisulphite-mediated sequencing of $5^{\prime}$ methylcytosine in genomic DNA. Nucleic Acids Res. 26: 50095010. doi: 10.1093/nar/26.21.5009.

Pontes, O., Li, C.F., Nunes, P.C., Haag, J., Ream, T., Vitins, A., Jacobsen, S.E., and Pikaard, C.S. 2006. The Arabidopsis chromatinmodifying nuclear siRNA pathway involves a nucleolar RNA processing center. Cell 126: 79-92.

Qi, Y., He, X., Wang, X.J., Kohany, O., Jurka, J., and Hannon, G.J. 2006. Distinct catalytic and noncatalytic roles of ARGONAUTE4 in RNA-directed DNA methylation. Nature 443: 1008-1012. 
Robb, G.B., Brown, K.M., Khurana, J., and Rana, T.M. 2005. Specific and potent RNAi in the nucleus of human cells. Nat. Struct. Mol. Biol. 12: 133-137.

Schwab, R., Ossowski, S., Riester, M., Warthmann, N., and Weigel, D. 2006. Highly specific gene silencing by artificial microRNAs in Arabidopsis. Plant Cell 18: 1121-1133.

Stam, M., Viterbo, A., Mol, J.N., and Kooter, J.M. 1998. Positiondependent methylation and transcriptional silencing of transgenes in inverted T-DNA repeats: Implications for posttranscriptional silencing of homologous host genes in plants. Mol. Cell. Biol. 18: 6165-6177.

Vaistij, F.E., Jones, L., and Baulcombe, D.C. 2002. Spreading of RNA targeting and DNA methylation in RNA silencing requires transcription of the target gene and a putative RNA-dependent RNA polymerase. Plant Cell 14: 857-867.

Waibel, F. and Filipowicz, W. 1990. RNA-polymerase specificity of transcription of Arabidopsis U snRNA genes determined by promoter element spacing. Nature 346: 199-202.

Wang, M.B. and Waterhouse, P.M. 2000. High-efficiency silencing of a $\beta$-glucuronidase gene in rice is correlated with repetitive trans- gene structure but is independent of DNA methylation. Plant Mol. Biol. 43: 67-82.

Wang, M.B., Li, Z.Y., Matthews, P.R., Upadhyaya, N.M., and Waterhouse, P.M. 1998. Improved vectors for Agrobacterium tumefaciens-mediated transformation of monocot plants. Acta Hortic. 461: 401-407.

Wang, M.B., Wesley, S.V., Finnegan, E.J., Smith, N.A., and Waterhouse, P.M. 2001. Replicating satellite RNA induces sequence-specific DNA methylation and truncated transcripts in plants. RNA 7: 16-28.

Watson, J.M., Fusaro, A.F., Wang, M.B., and Waterhouse, P.M. 2005. RNA silencing platforms in plants. FEBS Lett. 579: 5982-5987.

Xie, Z., Johansen, L.K., Gustafson, A.M., Kasschau, K.D., Lellis, A.D., Zilberman, D., Jacobsen, S.E., and Carrington, J.C. 2004. Genetic and functional diversification of small RNA pathways in plants. PLoS Biol. 2: 642-652. doi: 10.1371/journal. pbio.0020104.

Yu, J.Y., DeRuiter, S.L., and Turner, D.L. 2002. RNA interference by expression of short-interfering RNAs and hairpin RNAs in mammalian cells. Proc. Natl. Acad. Sci. 99: 6047-6052. 

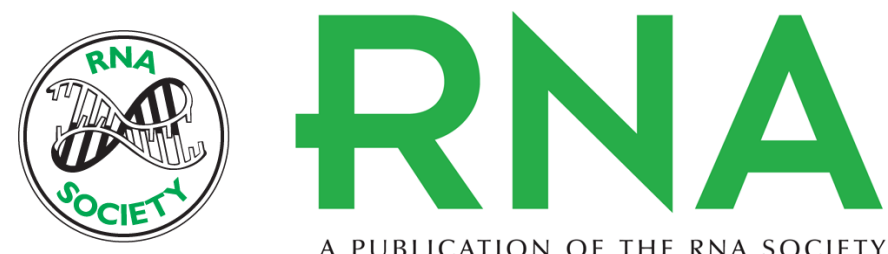

A PUBLICATION OF THE RNA SOCIETY

\section{Hairpin RNAs derived from RNA polymerase II and polymerase III promoter-directed transgenes are processed differently in plants}

Ming-Bo Wang, Christopher A. Helliwell, Li-Min Wu, et al.

RNA 2008 14: 903-913

Supplemental http://rnajournal.cshlp.org/content/suppl/2008/03/27/rna.760908.DC1

Material

References This article cites 34 articles, 10 of which can be accessed free at:

http://rnajournal.cshlp.org/content/14/5/903.full.html\#ref-list-1

License

Email Alerting Receive free email alerts when new articles cite this article - sign up in the box at the Service top right corner of the article or click here. 